\title{
Magnetic resonance imaging for the assessment of residual masses after treatment of non-Hodgkin's lymphomas
}

\author{
Carlos Montalbán, Jose Luis Rodriguez-Garcia, Luis Mazairas ${ }^{1}$, \\ Inmaculada Ayala ${ }^{1}$ and Jose Marcos-Robles ${ }^{1}$
}

\section{Departments of Internal Medicine and ${ }^{1}$ Radiology, Hospital Ramon y Cajal, Madrid, Spain}

\begin{abstract}
Summary: Eight patients with non-Hodgkin's lymphoma who were in clinical remission but showed residual masses after therapy were examined with magnetic resonance imaging at $1.5 \mathrm{~T}$ at the end of therapy, with the sequences TR/TE: 500/35, 500/70, 2000/35 and 2000/70. Residual masses were found in lymphoid areas (7), spleen (3) and kidney (1). In three patients, $T_{2}$ showed high signal intensity. Disease progressed in the following 1-3 months and active lymphoma was histologically identified. In the other five patients, $T_{2}$ showed a very low signal intensity. One of them had a distant relapse in lymphoid areas but the remaining four patients are asymptomatic after a mean follow-up time of 13 months, suggesting that the lymphoma is cured and the mass inactive.

We conclude that magnetic resonance imaging seems to distinguish active from non-active residual masses after treatment of non-Hodgkin's lymphoma patients otherwise in clinical remission.
\end{abstract}

\section{Introduction}

Residual masses can occasionally be found after completion of the initially planned treatment in Hodgkin's disease (HD) and non-Hodgkin's lymphoma (NHL), and their management is controversial. ${ }^{1,2}$ Surgical resection and subsequent histological examination is the most reliable method of evaluating the viability of tumour in these residual masses, but this procedure is not devoid of morbidity. As these masses may harbour no viable tumour cells, supplemental chemotherapy or radiotherapy should clearly not be given to the residual mass without pathological confirmation.

The usefulness of gallium scanning in treated patients for distinguishing active tumour from residual, presumed fibrotic masses is limited. ${ }^{3}$ Computed tomography (CT) and ultrasonography give valuable information of tumour size and distribution, but do not adequately reflect the nature of the mass (fibrosis, necrosis and inflammatory components). Thus, conventional radiology and CT are not able to determine whether the mass consists of active tumour or not. ${ }^{4,5}$ Fineneedle aspiration cytology of the mass provides diagnosis in some patients, but there are false negative results. ${ }^{6}$

Correspondence: Carlos Montalbán, M.D., Servicio de Medicina Interna, Hospital Ramón y Cajal, Carretera de Colmenar Km.9,100, 28034-Madrid, Spain.

Accepted: 14 January 1992
Previous reports have shown promising results with magnetic resonance imaging (MRI) in differentiating these conditions. ${ }^{5,7,8}$ The present study analysed the experience in treated patients with aggressive lymphoma who presented a radiologically detectable residual mass while otherwise appearing in a clinical complete remission (CR). We sought to correlate the residual masses and the clinical course with the findings in MRI in order to determine better whether MRI can be used to differentiate active from non-active disease.

\section{Patients and methods}

We studied eight patients with NHL who after treatment achieved a clinical remission, but in whom a residual mass of unknown significance was found on image studies. Patients were three men and five women (aged 22-67 years) (seven with high grade and the other with an intermediate grade NHL). Seven patients received only chemotherapy (MACOP-B: methotrexate with leucovorin rescue, doxorubicin, cyclophosphamide, vincristine, prednisone and bleomycin), and the involved nodes of another patients were treated only with radiation therapy. All patients in this study completed planned treatment and showed no evidence of persisting disease at restaging but the residual masses had been re-evaluated at the end of therapy both clinically by imaging with X-ray, ultrasound and CT scan. Persistent residual mass was defined 


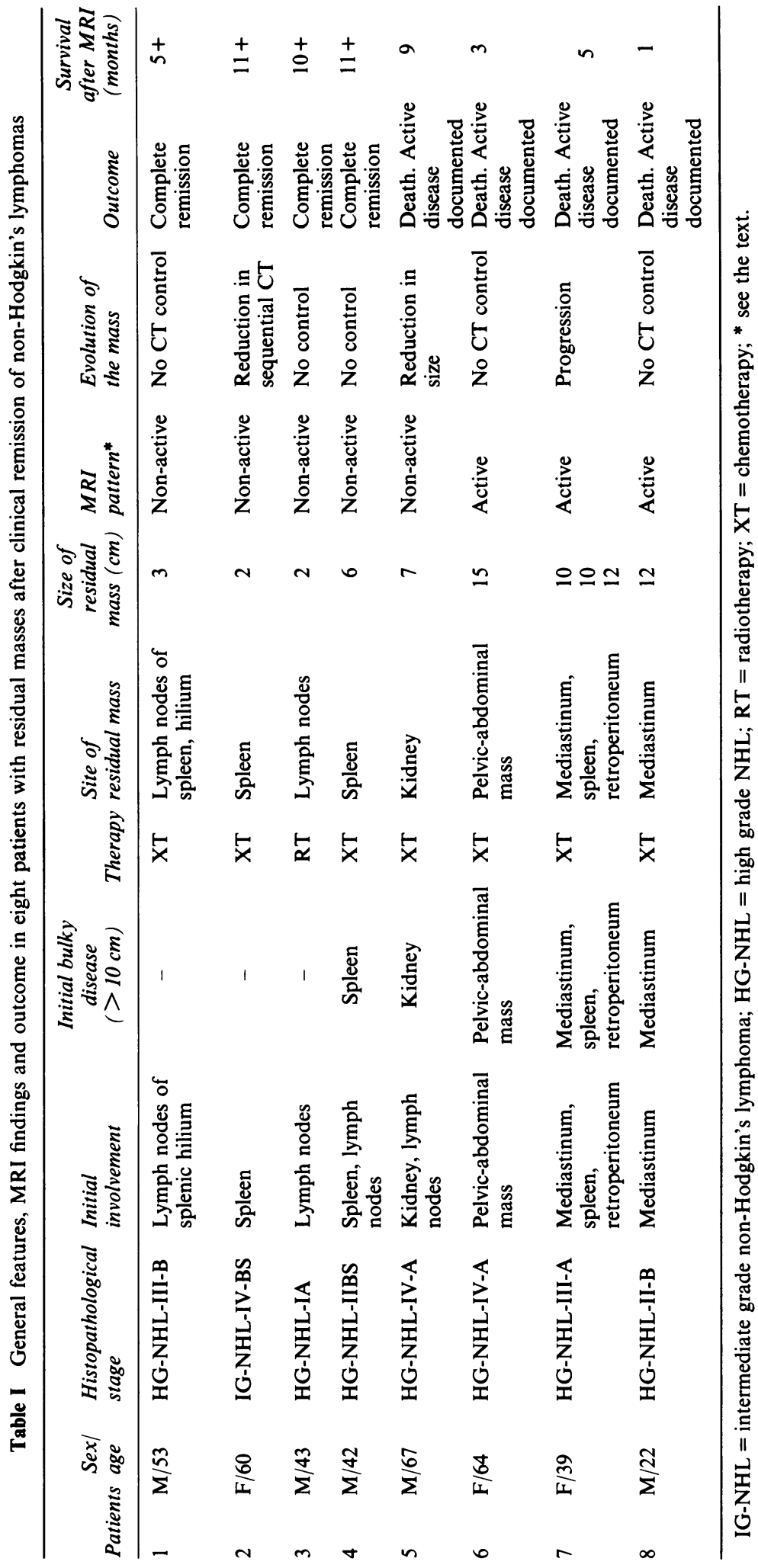


as any mass that being present before therapy, had initially responded to combination chemotherapy and/or radiation therapy and then remained stable in size after completed treatment. Masses were considered as bulky if they measured more than $10 \mathrm{~cm}$ in at least one diameter.

MRI was performed with a superconductive equipment (Siemens Magnetom, 1.5 T, software Numaris, version B1). Double echo, multi-slice, spin-echo techniques were used with repetition times (TR) of 500 and 2000 milliseconds and echo times (TE) of 35 and 70 milliseconds. In all patients, $T_{1}$ and $T_{2}$-weighted images were obtained in the transverse, axial and/or coronal planes. Contiguous, $10 \mathrm{~mm}$ sections were obtained in the 8 patients. A $256 \times 256$ matrix and a field of view of 450 were used in all cases. Photography, windowing and levelling of the images were automatically selected so that signal intensity could not be altered.

MRI analysis was made by two independent radiologists without knowledge of the final pathological and/or clinical diagnosis. Signal intensity of the lesion at the region of interest was evaluated and compared with striated muscle and adipose tissue signals. ${ }^{5}$
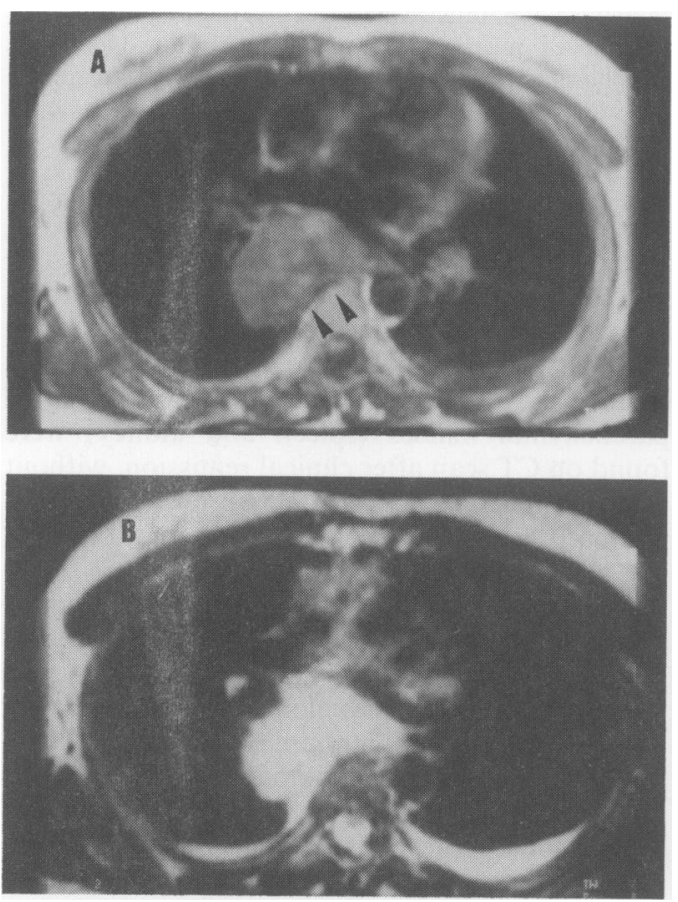

Figure 1 Patient 7. Transverse slices of the thorax. (A) Enlarged lymph nodes in the mediastinum (arrows) of a patient with non-Hodgkin's lymphoma having a low image intensity in $T_{1}$ (SE 500/35). (B) SE 2,000/70. The same mediastinal mass with high image intensity on $T_{2}$ suggesting active disease.

\section{Results}

Table I shows clinical characteristics, treatment results and outcome of the patients. The total follow-up period of patients varied between one and 11 months (average, 7 months) after MRI.

In three patients in whom large masses persisted, $T_{2}$-weighted images showed higher signal intensity than on $T_{1}$ : mediastinum in one case (case 8), mediastinum (Figure 1), spleen and retroperitoneum in another case (case 7) and pelvis and abdomen (Figure 2) in the remainder (case 6). Signal intensity was similar to that of fat. Disease progressed in the following 1-3 months and active lymphoma was pathologically documented on peripheral lymph nodes (two patients, Cases 7 and 8) and at laparotomy (one patient, Case 6, after genital bleeding). In the other five patients, on $T_{1}$ and $T_{2}$-weighted images, the lesions had a low signal intensity, similar to that of muscle (Figures 3 and 4, Cases 1 and 4). One of them (Case 5) had a distant relapse in lymphoid areas (cervical and supraclavicular areas) while residual mass within the kidney remained stable. The remaining four patients are asymptomatic for a mean of 13 months (range 9-15) after diagnosis. On follow-up, CT
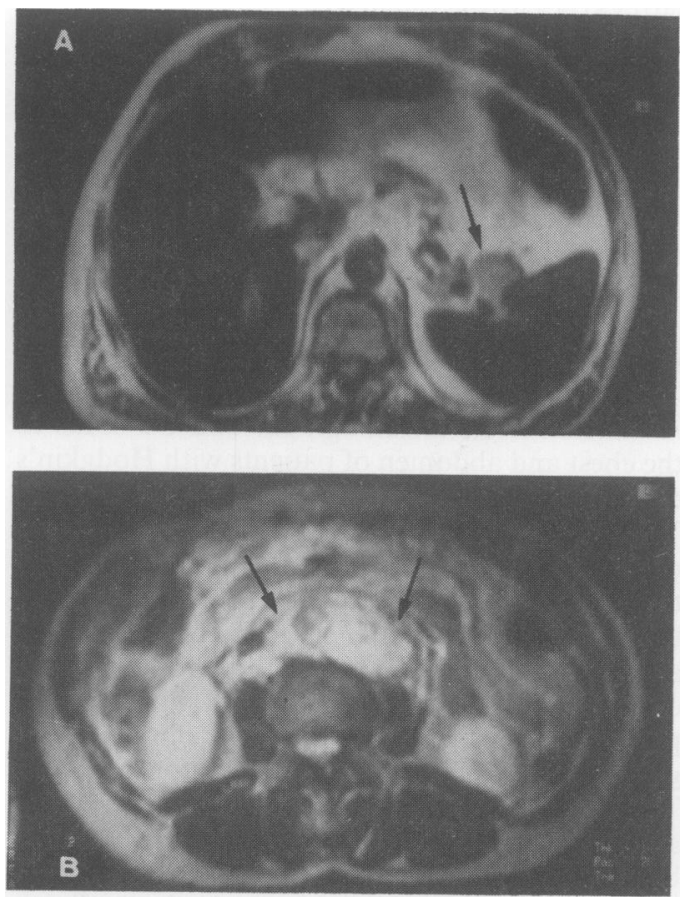

Figure 2 Patient 1. MRI of a patient with NHL that shows a low image intensity $T_{1}$-weighted image at the lymph nodes of the spleen hilium (arrows) in both $T_{1}(\mathrm{~A})$ and $T_{2}(\mathrm{~B})$ image intensities (non-active disease). 

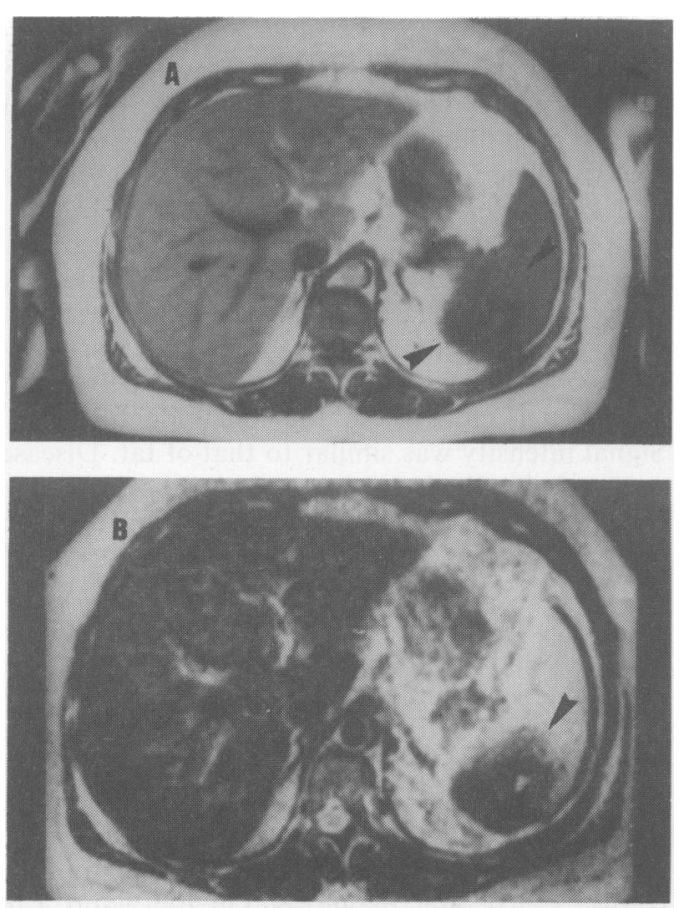

Figure 3 Patient 6. (A) A large retroperitoneal mass (arrows) in a patient with NHL $\left(T_{1}\right)$. (B) The mass shows a high-signal intensity on $T_{2}$ (arrow) suggesting active disease.

showed decrease in the size of the masses in two explored patients.

\section{Discussion}

A careful review of radiographs and CT scans of the chest and abdomen of patients with Hodgkin's disease show a high rate of residual abnormalities. The incidence is higher in those who present bulky masses, varying between $64 \%$ up to $88 \% .{ }^{9}$ In NHL, there are few references to residual masses proved to be non-malignant after surgery. However, when aggressive NHL is treated with third-generation chemotherapy regimens, the high incidence of persistent residual masses in patients in otherwise good clinical remission is an emerging problem. They have been found in $14-40 \%$ of the cases, appearing mainly in lymphoid areas with initial bulky disease. ${ }^{10-13} \mathrm{~A}$ few reports describe the histopathology of residual masses in the mediastinum and abdomen as a mixture of fibrosis, necrosis and inflammatory lesions. The proportion of different components in the masses are variable with a gradual increase of fibrosis after therapy. ${ }^{7}$ Fibrotic tissue has similar MRI characteristics as muscle
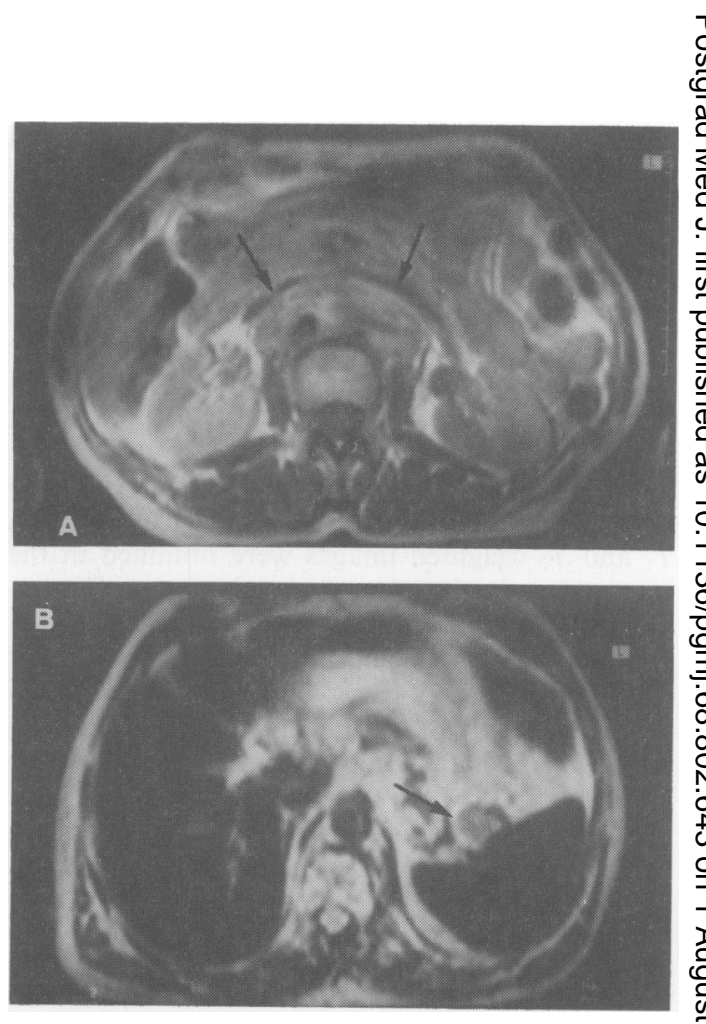

Figure 4 Patient 4. (A) $T_{1}$-weighted image $(500 / 35 \mathrm{~B} \vec{\bullet}$ showing a hypointense mass (arrow) in the spleen simila to that of muscle in a patient with non-Hodgkin's. lymphoma. (B) $T_{2}(2,000 / 70)$ showing the intrasplenic mass (arrow) with a very low signal intensity (non-activé disease).

and is displayed with low intensity in both $T_{\text {- }}$ and $T_{2}$-weighted images. ${ }^{5} \mathrm{We}$ have previously reported two patients who were treated with intensive chemotherapy and in whom large isolated intraparenchymal masses (spleen and kidney) were found on CT scan after clinical remission, without tumoral infiltration. ${ }^{14}$ In other series, viable lymphoma in a residual mass has been found only in $5 \%$ of patients. ${ }^{15}$

Recent studies with MRI suggest that no significant difference is discerned in the MRI parameters between lymph nodes with low- or high-grade $\mathrm{NHL}^{16-18}$ and that residual masses after treatment of non-Hodgkin's lymphomas might be distinguished from active lymphoma by the lower signal intensity of fibrosis on $T_{2}$-weighted images. ${ }^{19}$ Evaluation of these residual masses by MRI in NHL patients has been referred to previously. ${ }^{19-21}$

The data presented here show that two distinctive patterns on MRI, differentiating active from inactive lesions, seem to emerge when residual masses in patients with NHL in clinical remission are studied. When residual masses show a low signal intensity similar to that of muscle with all 
pulse sequences, the masses are probably composed of fibrotic material, as we have already shown. ${ }^{14}$ Residual masses from all patients who relapsed showed a low signal intensity on $T_{1^{-}}$ weighted images and high signal intensity on $T_{2}$-weighted images similar to that of fat, suggest-

\section{References}

1. Canellos, G.P. Residual mass in lymphoma may not be residual disease. J Clin Oncol 1988, 6: 931-933.

2. Uematsu, M., Kondo, M., Tsutsu, T. et al. Residual masses on follow-up computed tomography in patients with mediastinal non-Hodgkin's lymphoma. Clin Radiol 1989, 40: 244-247.

3. Peylan-Ramu, N., Haddy, T.B., Jones, E. et al. High frequency of benign mediastinal uptake of gallium-67 after completion chemotherapy in children with high-grade nonHodgkin's lymphoma. J Clin Oncol 1989, 7: 1800-1806.

4. Wernecke, K., Vassallo, P., Hoffman, G. et al. Value of sonography in monitoring the therapeutic response of mediastinal lymphoma: comparison with chest radiography and CT. $A J R$ 1991, 156: 265-272.

5. Nyman, R., Rehn, S., Glimelius, B. et al. Magnetic resonance imaging for assessment of treatment effects in mediastinal Hodgkin's disease. Acta Radiol 1987, 28: 145-151.

6. Zornoza, J., Cabanillas, F.F., Altoff, T.M. et al. Percutaneous needle biopsy in abdominal lymphoma. AJR 1981, 136: $97-103$.

7. Nyman, R., Rehn, S., Glimelius, B. et al. Residual mediastinal masses in Hodgkin's disease: prediction of size with MR imaging. Radiology 1989, 170: 435-440.

8. North, L.B., Fuller, L.M., Sullivan, J.A. \& Hagemeister, F.B. Regression of mediastinal Hodgkin's disease after therapy: evaluation of the time interval. Radiology 1987, 164: 599-602.

9. Radford, J.A., Cowan, R.A., Flanagan, M. et al. The significance of residual mediastinal abnormality on the chest radiograph following treatment for Hodgkin's disease. J Clin Oncol 1988, 6: 940-946.

10. Trédaniel, J., Lepage, E., Frija, J. et al. Les masses résiduelles après chimiothérapie des lymphomes non hodgkinienes. Nouv Rev Fr Hematol 1988, 30: 75-77.

11. Stewart, F.M., Williamson, B.R., Innes, D. \& Hess, Ch.E. Residual tumor masses following treatment for advanced histiocytic lymphoma. Cancer 1985, 55: 620-623. ing that the masses had been composed by active lymphoid tissue. MRI findings seem to be useful in the evaluation and management of residual masses occurring after remission in NHL, avoiding further unneccessary therapy in already cured patients.

12. Lewis, E., Bernardino, M.E., Salvador, P.G. et al. Posttherapy CT-detected mass in lymphoma patients. Is it viable tissue? J Comput Assist Tomogr 1982, 6: 792-795.

13. Montalbán, C., Rodríguez-García, J.L., Marcos-Robles, J. et al. Tratamiento de linfomas no Hodgkin de célula grande con protocolo MACOP-B. Med Clin (Barc) 1991, 97: 521-525.

14. Montalbán, C., Arechaga, S., Calleja, J.L. et al. Masas residuales no tumorales intraparenquimatosas después del tratamiento de linfomas no hodgkinianos de célula grande con quimioterapia intensiva. Med Clin (Barc) 1990, 94: 461-464.

15. Surbone, A., Longo, D.L., DeVita, V.T. et al. Residual abdominal masses in aggressive non-Hodgkin's lymphoma after combination chemotherapy. J Clin Oncol 1988, 6: $1832-1837$

16. Nyman, R., Rhen, S., Glimelius, B. et al. An attempt to characterize malignant lymphoma in spleen, liver and lymph nodes with magnetic resonance imaging. Acta Radiol 1987, 28: 527-533.

17. Rehn, S., Nyman, R., Glimelius, B. et al. Non-Hodgkin lymphoma: predicting prognostic grade with MR imaging. Radiology 1990, 176: 249-253.

18. Negendank, W.G., Al-Katib, A.M., Karanes, Ch. \& Smith, M.R. Lymphomas: MR imaging contrast characteristics with clinical-pathologic correlations. Radiology 1990, 177: 209216.

19. Zerhoumi, E.A., Fishman, E.K, Jones, R. et al. MR imaging of the "sterilized" lymphoma (abstract). Radiology 1986, 161: 207.

20. Markisz, J.A., Kovosky, P.A., Knowles, J.R. et al. MR imaging evaluation of response to therapy in lymphoma patients (abstract). Radiology 1988, 169: 175.

21. Drace, J., Carlsen, S. \& Chang, P. MR imaging strategies for differentiating benign residual versus active or recurrent tissue in lymphoma (abstract). Radiology 1989, 173: 273. 


\section{Erratum}

Re: Magnetic resonance imaging for the assessment of residual masses after treatment of non-Hodgkin's lymphomas. C. Montalban, J.L. Rodriguez-Garcia, L. Mazairas, I. Ayala and J. Marcos-Robles. Postgraduate Medical Journal 1992 68: 643-647.
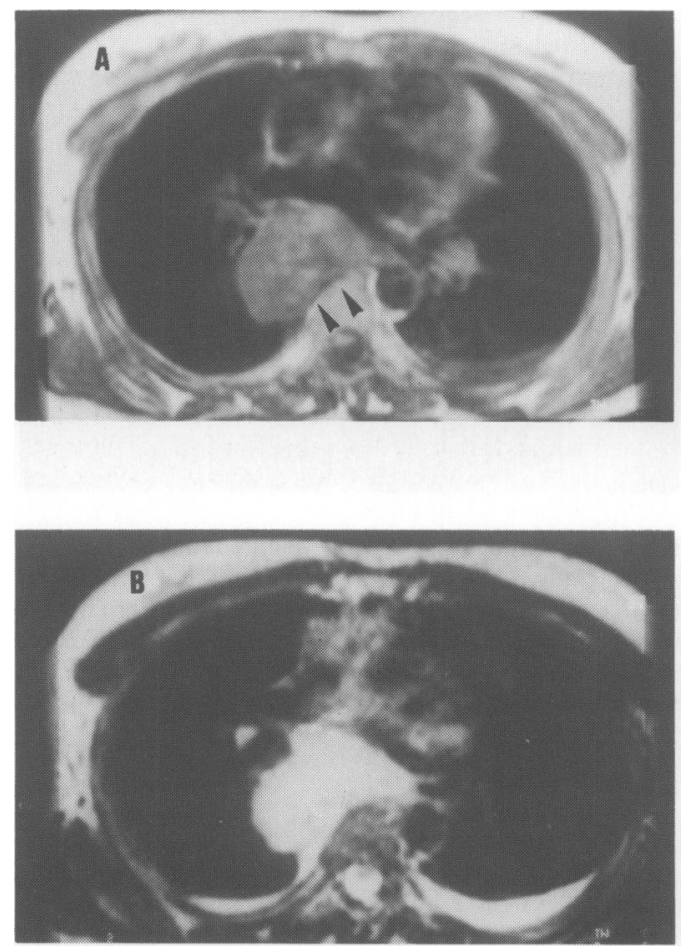

Figure 1 Patient 7. Transverse slices of the thorax. (A) Enlarged lymph nodes in the mediastinum (arrows) of a patient with non-Hodgkin's lymphoma having a low image intensity in $T_{1}$ (SE 500/35). (B) SE 2,000/70. The same mediastinal mass with high image intensity on $T_{2}$ suggesting active disease.

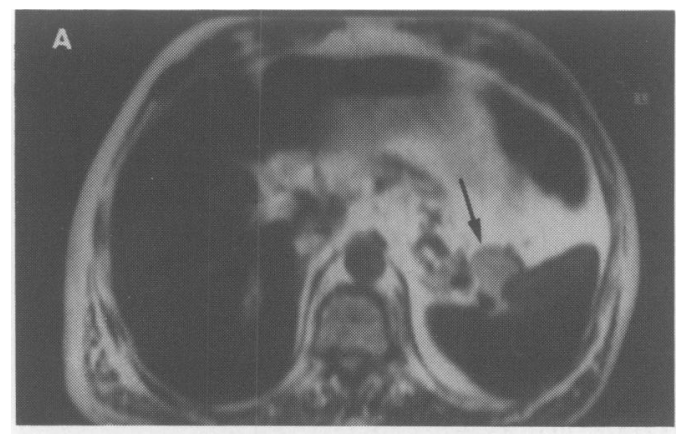

The Editorial Office and the authors apologise for incorrectly labelling the illustrations to the above article. We re-publish the illustrations

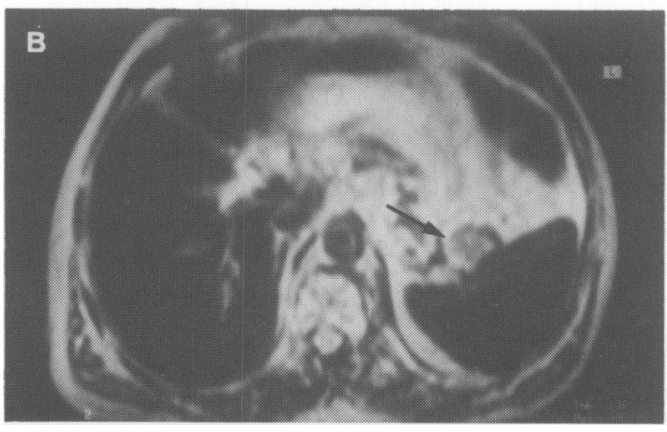

Figure 2 Patient 1. MRI of a patient with NHL that shows a low image intensity $T_{1}$-weighted image at the lymph nodes of the spleen hilium (arrows) in both $T_{1}(\mathrm{~A})$ and $T_{2}$ (B) image intensities (non-active disease). below, with the correct legends. 

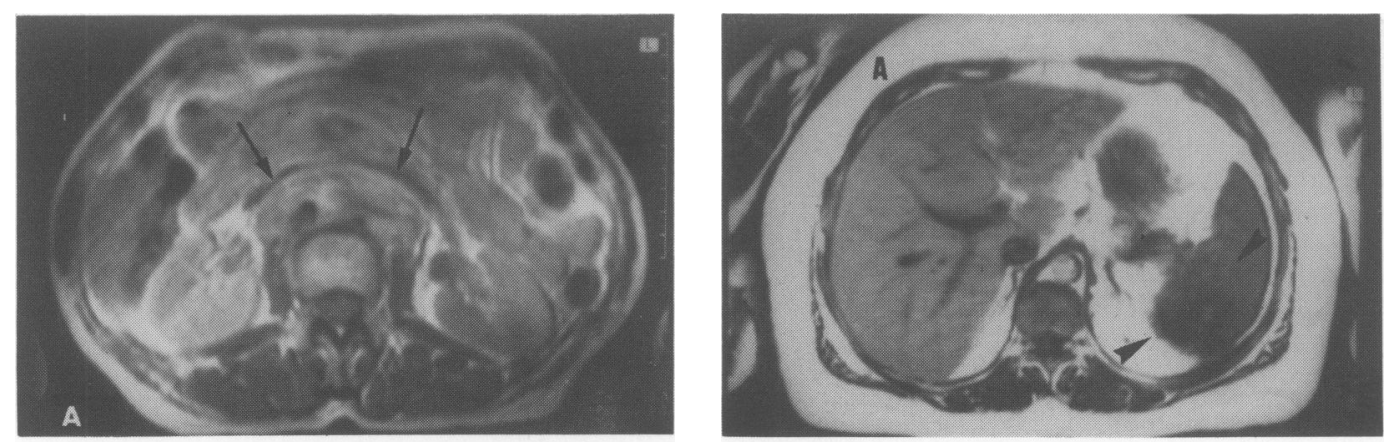

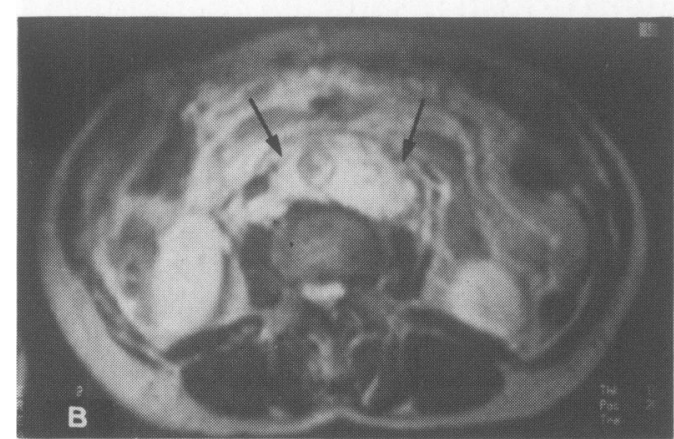

Figure 3 Patient 6. (A) A large retroperitoneal mass (arrows) in a patient with NHL $\left(T_{1}\right)$. (B) The mass shows a high-signal intensity on $T_{2}$ (arrow) suggesting active disease.

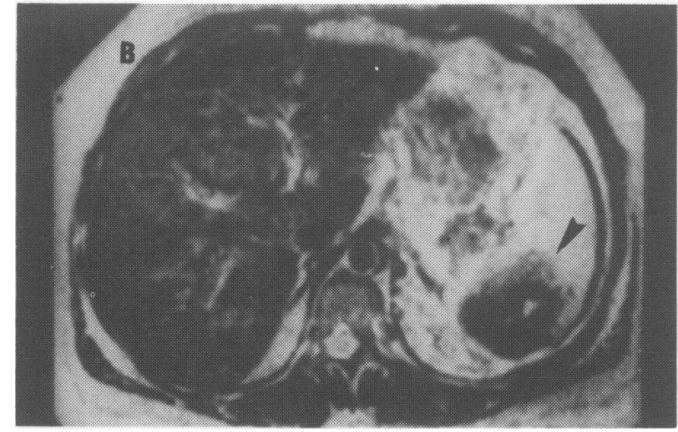

Figure 4 Patient 4. (A) $T_{1}$-weighted image (500/35) showing a hypointense mass (arrow) in the spleen similar to that of muscle in a patient with non-Hodgkin's lymphoma. (B) $T_{2}(2,000 / 70)$ showing the intrasplenic mass (arrow) with a very low signal intensity (non-active disease). 